\title{
DIFFERENTIABLY SIMPLE RINGS
}

\author{
EDWARD C. POSNER
}

Let $R$ be a ring and $D$ a family of derivations of $R$ into itself. We call $R$ differentiably simple under $D$ if $R^{2} \neq 0$ and if $R$ has no twosided ideal (other than 0 and $R$ ) sent into itself under every derivation of the family $D$ (i.e., has no differential ideal). We shall call $R$ differentiably simple, usually without specifying $D$. The purpose of this paper is to explore the analogy between simple rings and differentiably simple rings.

\section{Structure theory.}

TheOREM 1. Let $R$ be differentiably simple; then $R^{2}=R ;$ also, $R$ has no absolute left or right divisors of zero.

Proof. $R^{2}$ is a nonzero differential ideal, for every derivation. To prove the second part, let for example, $a R=0$, then $d(a R)=0$ $=d(a) R+a d(R)=d(a) R$ so $d(a) R=0$ so that the absolute left zero divisors are a (two-sided) differential ideal of $R$; since $R^{2} \neq 0$, this ideal is zero.

THEOREM 2. Let $R$ be differentiably simple and let $F$ be the set of those elements of the centroid of $R$ commuting with every derivation of $D$; then $F$ is a field, called the differential centroid of $R$; if $1 \in R, F$ is the subset of $R$ annihilated by every element of $D$.

Proof. $F$ is contained in the centroid, which is commutative since $R^{2}=R$; if $b$ is a nonzero element of $F, b(R)$ is a nonzero differential ideal of $R$, etc.

Lemma. $A$ differentiably simple ring $R$ is not locally nilpotent.

Proof. Consider for any $a$ not zero in $R$ the nonzero differential ideal consisting of all sums of two-sided multiples of all products of two derivatives of $a$ of any orders and mixture. This ideal is then the entire ring, so $a$ is in it. Let $n$ be the largest number of derivations occurring in one of the above-mentioned derivatives. We may suppose $n>0$. Consider the set of derivatives $d_{1}, \cdots, d_{k}$ which occur at all in the expression for $a$; differentiate this expression by every product $d_{i_{1}} d_{i_{2}} \cdots d_{i_{l}}, 0 \leqq l \leqq 2 n-1,1 \leqq i_{j} \leqq k, 1 \leqq j \leqq l$. Then $d_{i_{1}} d_{i_{2}} \cdots d_{i_{l}}(a)$ $=\sum r_{j_{1}} \cdots j_{q} d_{j_{1}} \cdots d_{j_{q}}(a) s_{j_{1}} \cdots j q$ for every $\left(i_{1}, \cdots, i_{l}\right), \quad 0 \leqq l$ $\leqq 2 n-1$ for every $1 \leqq i_{j} \leqq k$, the sum being extended over every

Presented to the Society, August 28, 1957; received by the editors June 30, 1959. 
$q$-tuple $\left(j_{1} \cdots j_{q}\right), 1 \leqq j_{q} \leqq k, 0 \leqq q \leqq 2 n-1$. Since the subring of $R$ generated by the $r_{j_{1}} \ldots j_{q}$ and $s_{j_{1} \ldots j_{q}}$ is nilpotent, by repeated use of this set of equations we find $a=0$ in contradiction to hypothesis.

Definitions. An ideal divisor of zero in a ring is a two-sided ideal annihilated by a nonzero element, on the right or on the left. A ring is called primary if every ideal divisor of zero is nilpotent.

\section{Theorem 3. A differentiably simple ring is primary.}

Proof. Suppose $I$ is a two-sided ideal of $R$ and $I b=0, b \neq 0$. We shall show that the Levitzki Nil Radical of $R$ contains a differential ideal if $I^{n}=0$ for no $n$. $a \in I \Rightarrow a x b=0 \forall x \in R$, so $d\left(a_{1}\right) x b+a_{1} x d(b)$ $=0 \forall x \in R, \quad d \in D, a_{1} \in R$. Then $a_{2}\left(d\left(a_{1}\right) x\right) b+a_{2} a_{1} x d(b)=0 \forall x \in R$, $a_{2} a_{1} x d(b)=0$. In general, $a_{n} a_{n-1} \cdots a_{1} x d_{1} d_{2} \cdots d_{n-1}(b)=0 \forall a_{1}, \cdots$, $a_{n} \in I, d_{1}, \cdots, d_{n} \in \mathcal{D}$. For $a \neq 0$, let $J=\{q / a x q=0 \forall x \in R\} . J$ is a two-sided ideal of $R$; we shall show that $J$ is locally nilpotent. For let $K=\left\{c \in R / c x \cdot \prod_{j=1}^{m} q_{i_{j}}=0, \forall x \in R\right.$, for all products of any length $m=m(c)$ chosen from a fixed finite set $\left.q_{1}, \cdots, q_{r}\right\} . K$ is a two-sided ideal of $R$, in fact a differential one (where $m(d(c))=2 m(c)$ ), and $K \neq 0$, so $K=R$. A fortiori, every product of length $t$ of the $q_{l}$ is zero, where $t=2+\max _{1 \leq l \leq r} m\left(q_{l}\right)$. That is, $J$ is locally nilpotent.

Since for any $n$ there is a product of $a_{i} \in I, a_{n} a_{n-1} \cdots a_{1}$ which is not zero, every mixed derivative of $b$ is in the Levitzki Nil Radical of $R$ (the maximal locally nilpotent ideal of $R$ ). $R$ itself would then be locally nilpotent, which contradicts the lemma. So $I^{n}=0$ for some $n$, which proves the theorem.

THEOREM 4. Let $R$ be a differentiably simple ring whose differential centroid is of characteristic zero. Then $R$ is a prime ring, i.e., there are no ideal divisors of zero.

Proof. We prove more generally that if $R$ is a primary ring whose additive group is torsion-free, and $a$ lies in a nilpotent ideal, then $d(a)$ lies in a nilpotent ideal, for every derivation $d$ of $R$. So let $n$ be the smallest integer such that $a x_{1} a x_{2} \cdots a x_{n}=0 \forall x_{i} \in R, 1 \leqq i \leqq n$. Assume $d(a)$ does not lie in a nilpotent ideal. Differentiate the preceding equation $n$ times, and left multiply by $a t_{1} a t_{2} \cdots a t_{n-1}$, where $t_{j}$, $1 \leqq j \leqq n-1$ are any elements of $R$. We obtain $(n+1) a t_{1} a t_{2} \ldots$ $a t_{n-1} d(a) x_{1} d(a) x_{2} \cdots d(a) x_{n}=0, \quad a t_{1} a t_{2} \cdots a t_{n-1} d(a) x_{1} d(a) x_{2} \cdots$ $d(a) x_{n}=0 \forall t_{j} \in R, 1 \leqq j \leqq n-1, \forall x_{i} \in R, 1 \leqq i \leqq n$. For simplicity, right multiply by $d(a)$; then $d(a)$ lies in an ideal divisor of zero and hence in a nilpotent ideal, or else $a t_{1} a t_{2} \cdots a t_{n-1} d(a) x_{1} \cdots x_{n-1} d(a)=0$. This latter case must occur. Continuing this stripping off process, we find $a t_{1} a t_{2} \cdots a t_{n-1} d(a)=0, \forall t_{1}, \cdots, t_{n-1} \in R$. Then $d(a)$ lies in 
an ideal divisor of zero, which is impossible, or else $a t_{1} a t_{2} \cdots a t_{n-1}$ $=0 \forall t_{1} \cdots t_{n-1} \in R$, which contradicts the minimality of $n$.

Then every differentiably simple ring of characteristic zero is prime. For every ideal divisor of zero is nilpotent, so that if $R$ has no nilpotent ideals, we are done; if $R$ has, however, a nilpotent ideal, we have just shown that the sum of the nilpotent ideals of $R$ is a differential ideal, hence all of $R$. Then $R$ would be locally nilpotent, which we know to be impossible.

For an application of this result to algebraic functions see [1].

COROLlaRY. A differentiably simple ring of characteristic zero with a minimal two-sided ideal is simple. In particular if $R$ satisfies the descending chain condition for left or right ideals, $R$ is simple.

Proof. Let $R$ be differentiably simple, and $I$ a minimal 2 -sided ideal $\neq 0$. $I^{2} \neq 0$ since $R$ is prime, so $I^{2}=I$. Then $I$ is differential (for any derivation), so $I=R, R$ is simple.

THEOREM 5. Every commutative differentiably simple ring has a unit.

Proof. We must separate the characteristic $p$ and zero cases. First let $R$ be differentiably simple under $D$ and of characteristic $p \neq 0$. For every $x \in R, d \in D, d\left(x^{p}\right)=0$, so (here is where we use commutativity) $x^{p}$ is in the differential centroid of $R$. If every $x^{p}=0$, then $R$ is a commutative nil ring, hence locally nilpotent. But a differentiably simple ring is not locally nilpotent. Hence for at least one $x \in R, x^{p} \neq 0$ and $R$ contains a nonzero element of the differential centroid, and hence a unit.

Now let $R$ be commutative differentiably simple and of characteristic zero. We shall assume that $D=\{d\}$, but only to simplify notation. Look at the proof of the lemma in Theorem 3. Let $a$ be any nonzero element of $R$. Then there are elements $r_{i j} \in R$ such that $a=\sum_{i=0 ; j=0}^{n} \sum^{n} r_{i j} a^{(i)} a^{(j)}$. Differentiate $2 n-1$ times (without loss of generality, we can assume $n>0)$. Then $a^{(k)}=\sum_{i=0}^{2 n-1} t_{k, i} a^{(i)}, 0 \leqq k$ $\leqq 2 n-1, t_{k, i} \in R$. Consider the column vector with $2 n$ components, $v$, whose $j$ th component is $a^{(2 j-1)}$, and the matrix $T=\left(t_{k, i}\right)$. Then $v=T v$. Regard $T$ as a matrix over the quotient field of $R$ ( $R$ has no divisors of zero since it is a commutative prime ring). $(T-I) v=0$. But $v \neq 0$ since $a \neq 0, T-I$ is singular.

So $\operatorname{det}(T-I)=0$. Expand this out. Every term except the product of the 1 's on the diagonal contains at least one element of $R$ and the rest 1 's. Then $r-(-1)^{2 n} \in R$ where $r \in R$ so the unit 1 of the quotient field actually appears as an element of $R, R$ has a unit. 


\section{Extensions of differentiably simple rings.}

THEOREM 6. Let $R$ be a commutative ring differentiably simple under $D$ with differential centroid $F$. Let $M$ be a nonempty multiplicitively closed subset of $R$ containing 1 but not zero. Let $R_{M}$ be the ring of quotients of $R$ with respect to $M$. Let $D_{M}$ be the set of derivations of $D$ uniquely extended to $R_{M}$. Then $R_{M}$ is differentiably simple under $D_{M}$ with differential centroid $F$.

Proof. Let $I$ be a nonzero differential ideal of $R_{M}$; then the set of $a$ in $R$ for which $\exists b \in M$ with $a / b \in I$ is a nonzero differential ideal of $R$ contained in $I$. So $R \subset I, I=R_{M}$. To prove $R_{M}$ has differential centroid $F$, we invoke the following lemma.

Lemma. A commutative differentiably simple ring $R$ is differentially closed in its full ring of quotients $S$.

Proof. We are to prove that if $a \in S$ and $d(a) \in R \forall d \in D$, then $a \in R$. Let $J=\{x \in R / x a \in R\} . J$ is a differential ideal of $R$ and is not zero. Then $J=R, 1 \cdot a \in R, a \in R$.

Returning to the theorem, we are to prove that if $d(a)=0 \forall d \in D$, then $a \in F$. But by the Lemma, $a \in R$, whence $a \in F$.

As an application of this theorem, consider a differential field $L$. (The differential centroid of a differential field is also called its field of constants.) We wish to prove the known result from differential algebra that an integral can be adjoined to a differential field with the addition of no new constants. Consider $L$ a differential field, $x$ a differential indeterminate over $L$, and, in the field $L(x)$, extend the original derivation by $x^{\prime}=a$, where $a$ was not a derivative in $L$. We wish to show that $L(x)$ has no new constants. However, it can be shown that $L[x]$ is differentiably simple and has the same differential centroid as $L$. The quotient field $L(x)$ of $L[x]$ has the same differential centroid as $L[x]$, and hence the same as $L$. That is, $L(x)$ has no new constants.

Theorem 7. Let $R$ be differentiably simple under $D$ with differential centroid $F$. Let $K$ be a field containing $F$. Then $R \otimes_{F} K$ (under the natural extension of $D)$ is differentiably simple, with differential centroid $K$.

Proof. Consider the subring of the full ring of $F$-endomorphisms of $R$ generated by left and right multiplications by elemerrts of $R$ and by differentiation by elements of $D$. To say that $R$ is differentiably simple under $D$ is the same as saying that $R$ is irreducible under this ring. Furthermore, the differential centroid is just the commuting ring of this ring of endomorphisms. The tensor product 
over $F$ of this ring and $K$ is the analogous ring for $R \otimes_{F} K$. Reference [2] is just what we need to say that $R \otimes_{F} K$ is irreducible under this tensored ring with commuting ring $K$, i.e., differentiably simple with differential centroid $K$.

3. The ring of differential polynomials. We are going to study the above ring of endomorphisms when $R$ is commutative. First consider the ring of differential polynomials over $D$ with coefficients in $R$, $A_{\mathscr{D}}(R)$. This will be the ring of polynomials in noncommuting indeterminates, one for each derivation in $D$, with coefficients from $R$ written on the left. Here multiplication is defined by $D a=d(a)+a D$ where $D$ is the indeterminate corresponding to the derivation $d \in D$. Then $A_{\mathscr{D}}(R)$ is an (associative) ring, and what is more, $R$ is a left $A_{\mathscr{D}}(R)$ module by $D(a)=d(a) . R$ is contained in $A_{\mathscr{D}}(R)$ as polynomials of degree zero. Each $d \in D$ extends to the inner derivation of $A_{\mathscr{D}}(R)$ given by $x \rightarrow D x-x D . R$ is an irreducible left $A_{D}(R)$ module if and only if $R$ is differentiably simple under $D$. Let $B_{\mathscr{D}}(R)$ be $A_{\mathscr{D}}(R)$ made faithful on $R$. (This is the ring of the preceding section.) $R$ is still contained in $B_{\mathscr{D}}(R)$ since $R$ has no absolute left divisors of zero. $F$ is the commuting ring of endomorphisms of $B_{\mathfrak{D}}(R)$ acting on $R$, and also of $A_{\mathscr{D}}(R)$ acting on $R$. Let $N$ be the ideal of $A_{\mathscr{D}}(R)$ annihilating $R$, so that $A_{\mathscr{D}}(R) / N=B_{\mathscr{D}}(R) . B_{\mathscr{D}}(R)$ is left primitive, $R$ being a faithful irreducible left module.

THEOREM 8. Let $R$ be a commutative differentiably simple ring of characteristic $p(\neq 0$ forced $)$ of finite dimension over its differential centroid $F$. Then $B_{D}(R)$ is the full ring of F-linear transformations of $R$ into itself. If $R \neq F,[R: F] \equiv O(p) ;$ if $D$ contains but one derivation, $[R: F]$ is a power of $p$.

Proof. $B_{D}(R)$ is the full ring of $F$-linear transformations of $R$ into itself, by the density theorem. Let $R \neq F$, so that $D$ contains a nonzero derivation. Consider $\{d(a), d \in D, a \in R\}$ : there is a non-nilpotent element in this set, since $R$ is not nil. Let $d(a)$ be not nilpotent. Let $D$ be the indeterminate corresponding to $d$. Then $(D a-a D)^{p}$ $=(d(a))^{p}=\lambda \in F$, and $\lambda$ is a nonzero commutator in $B_{\mathscr{D}}(R)$, namely $D\left(a(D a)^{p-1}\right)-\left(a(D a)^{p-1}\right) D$. The trace of $\lambda$ is zero, $[R: F] \equiv O(p)$. If $D=\{d\}$, let $k$ be the least degree of elements of $N$. Let $J(\subset R)$ be the set consisting of zero and coefficients of $D^{k}$ for some element of degree $k$ in $N . J$ is an ideal of $R$, and if $a_{0}+a_{1} D+\cdots+a_{k} D^{k} \in N$, $D\left(a_{0}+a_{1} D+\cdots+a_{k} D^{k}\right)-\left(a_{0}+a_{1} D+\cdots+a_{k} D^{k}\right) D \in N$ and equals $\cdots+(\cdots) D^{k-1}+d\left(a_{k}\right) D^{k}$, so that $J$ is a nonzero differential ideal of $R$. Hence $J$ contains an element $b_{0}+b_{1} D+\cdots+b_{k-1}$ 
$+D^{k}$. Let $m=[R: F]>1 . \operatorname{dim}_{F} A_{D}(R) / N=m^{2}$ by the first part of the theorem, but now $\operatorname{dim}_{F} A_{D}(R) / N \leqq k m$ so $m \leqq k$. Since $D$ has a characteristic polynomial of degree $\leqq m, k \leqq m, k=m$. (Then $b_{0}$, $b_{1}, \cdots, b_{k-1} \in F$ and $X=b_{0}+b_{1} D+b_{m-1} D^{m-1}+D^{m}$ is the characteristic and minimal polynomial for $D$.) For all $a \in R, X a-a X \in N$ and is of degree less than $m$, hence is zero.

$$
\begin{aligned}
X a-a X= & \left(X-b_{0}\right)(a)+\cdots+\left[b_{m-1} C_{m-1,1} d(a)+C_{m, 2} d^{2}(a)\right] D^{m-2} \\
& +C_{m, 1} d(a) D^{m-1} .
\end{aligned}
$$

Then

$$
C_{m, 1} D=0, C_{m, 1} \equiv O(p) ; \quad b_{m-1} C_{m-1,1} D+C_{m, 2} D^{2}=0,
$$

in particular,

$$
C_{m, 2} \equiv O(p), \cdots, C_{m, m-1} \equiv O(p) .
$$

So all nontrivial binomial coefficients of $m$ are multiples of $p, m$ is a power of $p$, as promised.

We have seen that $B_{\mathscr{D}}(R)$ is simple if $R$ is finite dimensional over its differential centroid; it is always simple.

THEOREM 9. If $R$ is a commutative ring differentiably simple under $D$ with differential centroid $F$, then $B_{D}(R)$ is simple with centroid $F$.

Proof. Obviously $\left(B_{\mathscr{D}}(R)\right)^{2} \neq 0$. We must show that if $I$ is a twosided ideal of $A_{\mathscr{D}}(R)$, then either $I$ is contained in $N$, or $I+N$ $=A_{D}(R)$. Let $x$ be a nonzero element of $I$ whose degree is minimal with respect to having a nonzero constant term $a$ (if any such exist at all). Then for all $b$ in $R, x b-b x \in I$, is of degree less than $x$, and has as constant term $(x-a)(b)$. Then $(x-a)(b)=0$ for all $b \in R$, $x-a \in N, I+N$ contains the nonzero element $a$ of $R$. For all $d \in \mathbb{D}$, $D a-a D \in I+N, d(a) \in I+N,(I+N) \cap R$ is a nonzero differential ideal and contains $1, I+N=A_{\mathscr{D}}(R)$. If on the other hand every element of $I$ has zero constant term, then for all $b \in R, x b-b x \in I$ with constant term $x(b)$. Then $x(b)=0, x \in N$ if $x \in I, I \subset N$.

The above paragraph proves that no element of $N$ has nonzero constant term, for otherwise $A_{\mathscr{D}}(R)=N$. Let $\alpha$ be in the centroid of $B_{\mathfrak{D}}(R)$ and $a, b$ be in $R . \alpha(a b)=\alpha(a) b, \alpha(b a)=b \alpha(a)$, so $\alpha(a)$ commutes with elements of $R$. If $y$ in $A_{\triangleright}(R)$ is $\alpha(a)(N)$, then $y b-b y \in N \forall b \in R$. But $y b-b y$ has constant term $\left(y-y_{0}\right)(b)$, where $y_{0}$ is the constant term of $y$. So $\left(y-y_{0}\right)(b)=0 \forall b \in R, y-y_{0} \in N, \alpha(a) \in R$ in $B_{\mathscr{D}}(R)$ (being $\equiv(\bmod N)$ to $\left.y_{0}\right)$. That is, by abuse of language, $\alpha$ is in $R$, since $R$ has a unit. The proof that $d(\alpha)=0 \forall d \in D$ is straightforward and omitted. 
REMARK. If $F$ is a commutative differential ring with no absolute divisors of zero such that $B_{\mathfrak{D}}(R)$ is simple, $R$ is differentiably simple. The proof is similar.

THEOREM 10. Let $R$ be a commutative differentiably simple ring of characteristic zero such that the elements of $D$ commute with one another. Let $C_{\mathscr{D}}(R)$ be the ring of differential polynomials over $R$ in commuting indeterminates. Then $C_{D}(R)$ is simple if and only if the elements of $D$ are left linearly independent over $R$.

The proof is left to the reader.

\section{BIBLIOGRAPHY}

1. E. C. Posner, Integral closure of differential rings, Pacific J. Math., to appear.

2. T. Nakayama and G. Azuyana, On irreducible rings, Ann. of Math. vol. 48 (1947) p. 956.

UNIVERSITY OF WISCONSIN 